\title{
SUSY naturalness without prejudice
}

\author{
D. M. Ghilencea \\ Theoretical Physics Department, National Institute of Physics \\ and Nuclear Engineering (IFIN-HH) Bucharest, P.O. Box MG-6 077125, Romania \\ CERN Theory Division, CH-1211 Geneva 23, Switzerland
}

(Received 12 March 2014; published 8 May 2014)

\begin{abstract}
Unlike the Standard Model (SM), supersymmetric models stabilize the electroweak (EW) scale $v$ at the quantum level and predict that $v$ is a function of the TeV-valued SUSY parameters $\left(\gamma_{\alpha}\right)$ of the UV Lagrangian. We show that the (inverse of the) covariance matrix of the model in the basis of these parameters and the usual deviation $\delta \chi^{2}$ (from $\chi_{\min }^{2}$ of a model) automatically encode information about the "traditional" EW fine-tuning measuring this stability, provided that the EW scale $v \sim m_{Z}$ is indeed regarded as a function $v=v(\gamma)$. It is known that large EW fine-tuning may signal an incomplete theory of soft terms and can be reduced when relations among $\gamma_{\alpha}$ exist (due to GUT symmetries, etc.). The global correlation coefficient of this matrix can help one investigate if such relations are present. An upper bound on the usual EW fine-tuning measure ("in quadrature") emerges from the analysis of the $\delta \chi^{2}$ and the $s$-standard deviation confidence interval by using $v=v(\gamma)$ and the theoretical approximation (loop order) considered for the calculation of the observables. This upper bound avoids subjective criteria for the "acceptable" level of EW fine-tuning for which the model is still "natural."
\end{abstract}

DOI: $10.1103 /$ PhysRevD.89.095007

PACS numbers: 12.60.Jv, 12.60.-i

\section{A NATURAL TEST FOR SUSY MODELS}

\section{A. Introduction}

Unlike the Standard Model (SM), supersymmetric models (MSSM, NMSSM, etc.) stabilize the electroweak (EW) scale $v$ at the quantum level and make a prediction for it. The combined Higgses EW VEV $v$, or the $Z$ boson mass $m_{Z} \propto v$, is a derived quantity that is a function $v=v(\gamma)$, where $\gamma_{\alpha}$ denote the following Lagrangian UV parameters: the TeV-valued soft masses, soft couplings, and $\mu$. The function $v=v(\gamma)$ is obtained from the minimization of the Higgs potential and from the fact that the Higgs couplings are fixed, to lowest order, by gauge interactions (unlike the SM case where the Higgs self-coupling is arbitrary). Whether this prediction successfully recovers the measured value $m_{Z}^{0} \approx 91.187 \mathrm{GeV}$ of the $Z$ boson is a natural test of SUSY. This regards $m_{Z}$ as an observable to be fitted. This view, adopted here, remains true to the original motivation of SUSY.

This naturalness test received much attention from theorists who long ago introduced fine-tuning [1] measures [2,3] for it. However, precision data fits [4] often prefer to keep $m_{Z}$ as a fixed input (constant) equal to $m_{Z}^{0}$ rather than as an observable as well that depends on the Lagrangian parameters and then no $\chi^{2}$ "cost" to fit $m_{Z}$ is usually reported. ${ }^{1}$

\footnotetext{
*dumitru.ghilencea@cern.ch

${ }^{1}$ This is partly due to technical and historical reasons from preSUSY fits where $m_{Z}$ is an input; with $m_{Z}$ output also the parameter scans would be very ineffective, as many points are ruled out by $Z$ mass.
}

At the same time, some data fits still report the EW finetuning in which $v \sim m_{Z}$ is indeed a function $v=v(\gamma)$, often giving a large variation of $m_{Z}$ (about its fixed input value). It is not clear to us how results relying on two different assumptions [ $v$ fixed constant or a function $v=v(\gamma)$ ] can be combined consistently to draw a clear conclusion. This is due to the following questions (particularly $Q_{3}$ below):

$Q_{1}$ : Is the likelihood to fit the observable $m_{Z}$ related to the EW fine-tuning "cost"?

$Q_{2}$ : What is the link of the total likelihood to fit a set of observables to EW fine-tuning?

$Q_{3}$ : How do we compare a model with a good fit (of $m_{Z}$ and other data) and "large" EW fine-tuning to one with nearly as good a fit but less fine- tuning (for the same data)?

Question $Q_{1}$ is even more compelling given that we do not know what an "acceptable" value of the EW fine-tuning is. One can address $Q_{1,2,3}$ by regarding $m_{Z}$ as an observable and by using standard tools to test models, as discussed in the likelihood approach $[5,6]$ or earlier in the Bayesian case [7-9]. These works suggested that EW fine-tuning is related to the likelihood $\left(\chi^{2}\right)$ or the posterior probability to fit the data that includes the observable $m_{Z}$. In this letter we explore this relation further, using a different approach, and $\mathrm{a} \chi^{2}$ (frequentist) analysis. As detailed below, we study a possible relation of the covariance matrix of the model to the EW fine-tuning. This connection is not examined in the literature even though each of these aspects were studied in the past separately. This is the main purpose of this work.

As an example, consider the MSSM case with the Higgs potential minimum conditions in a standard notation, fixing the EW scale $\left(v \propto m_{Z}\right)$ and $\tan \beta$ (or $B$ ), 


$$
\begin{aligned}
\frac{m_{Z}^{2}}{2} & =-\mu^{2}+\frac{m_{1}^{2}-m_{2}^{2} \tan ^{2} \beta}{\tan ^{2} \beta-1}+\ldots \\
2 m_{3}^{2} & =\left(m_{1}^{2}+m_{2}^{2}+2 \mu^{2}\right) \sin 2 \beta+\cdots .
\end{aligned}
$$

The dots stand for quantum corrections to the quartic Higgs couplings and $m_{1,2,3}$ are one-loop soft masses. In precision data fits, one traditionally replaces $m_{Z}$ "by hand" by its measured mass $m_{Z}^{0}$ to fix $\mu$ instead (as a function of remaining $\gamma_{\alpha}$ ), or "fine-tune" the independent $\gamma_{\alpha}$ to reproduce $m_{Z}^{0}$. Ultimately, this amounts to using a Dirac $\delta$ distribution for the observable $m_{Z}$. Further [5,6],

$$
\begin{aligned}
\delta\left(1-m_{Z} / m_{Z}^{0}\right) & =\frac{1}{\Delta} \delta\left[n^{\alpha}\left(1-\gamma_{\alpha} / \gamma_{\alpha}^{0}\right)\right], \\
\gamma & =\left\{m_{0}, \mu, A_{0}, B_{0}, M_{1}, M_{2}, M_{3}, \ldots\right\} .
\end{aligned}
$$

Here $m_{Z} \sim v$ is a function of parameters $\gamma_{\alpha}$ as shown in Eq. (1), with $\gamma_{\alpha}^{0}$ components of the set $\gamma$ that respect the condition $m_{Z}\left(\gamma_{\alpha}^{0}\right)=m_{Z}^{0}$ and $n^{\alpha}$ are the components of the normal to the surface defined by this equation; finally,

$$
\Delta \equiv\left\{\sum_{\alpha}\left(\frac{\partial \ln m_{Z}(\gamma)}{\partial \ln \gamma_{\alpha}}\right)_{\gamma=\gamma^{0}}^{2}\right\}^{1 / 2} .
$$

Since $\Delta$ emerged from fixing the EW scale condition $\left(m_{Z}=m_{Z}^{0}\right)$ associated with fine-tuning, we can only interpret it as a derived, unique measure of fine-tuning (not chosen).

The message is that the distribution in the lhs of Eq. (2) chosen as a likelihood (for observable $m_{Z}$ ) somehow "knows" about the EW fine-tuning $\Delta$. This may not be too surprising, but it hints to a deeper connection. The EW scale $v=v(\gamma)$ enters in many observables and also correlations among these can be present. Therefore, they can also have significant individual fine-tunings associated. We usually refer to fine-tuning of $m_{Z}$, but one can similarly discuss, for example, the fine-tuning of the Higgs mass, etc, since this is also closely related to the hierarchy problem. Then a relation similar to Eq. (2) can be present between each observable and the set of parameters $\gamma$. This seems to suggest an underlying connection of the likelihood (or usual $\chi^{2}$ ) associated with a set of EW observables to their fine-tuning and to the distribution of the parameters of the model about their central values (of maximal likelihood or $\min \chi^{2}$ ); these could be connected as in the example above, by some general form of $\Delta$. These comments indicate an affirmative answer to question $Q_{1}$. For a more detailed discussion see $[5,6]$.

In the following we explore some of these issues further. We consider that

(1) in practice one does not have Dirac distributions for $m_{Z}$ or other observables,

(2) correlations can exist between $m_{Z}$ and other observables: $m_{h}, m_{H}$, etc.,

(3) and other observables can also depend on the EW scale $v=v(\gamma)$ (and/or on $\gamma$ ).
With these in mind we study the link of the likelihood to fit the data and its deviation from the maximal value, to the EW fine-tuning ( $\Delta$ above); equivalently, in a chi-square language, we study the link of the deviation $\delta \chi^{2}$ from the minimal value $\chi_{\min }^{2}$, to the EW fine-tuning, $\Delta$. We find that, just as the likelihood to fit $m_{Z}^{0}$ contains $\Delta$ [Eq. (2)], in the general case [with (1), (2), (3)] of the total likelihood, EW fine-tuning is automatically present in the covariance matrix $\tilde{M}$ in the basis of the fundamental parameters $\left(\gamma_{\alpha}\right)$ and thus also in the deviation $\delta \chi^{2}$, provided that we regard $v$ as a function $v=v(\gamma)$, (as predicted by SUSY). Thus the matrix $\tilde{M}$ has a more fundamental role than the EW fine-tuning and contains information about the stability of the EW scale under UV variation of SUSY parameters. This view also ends a long-held distinction between EW fine-tuning (to fit $\left.m_{Z}\right)$ and that to fit other observables $\left(m_{h}, m_{H}\right.$, etc) that also depend on $v$ and that are thus ultimately linked to the hierarchy problem (just as $m_{Z, W}$ are).

\section{B. The link of the likelihood $\left(\chi^{2}\right)$ to EW fine-tuning}

Consider a model with a number of observables $\mathcal{O}_{i}$ $(i=1,2, \ldots, n)$ of central experimental values $\mathcal{O}_{i}^{0}$ fitted using a set of SUSY parameters ${ }^{2} \gamma_{\alpha},(\alpha=1,2 . ., s)$ that enter in the Lagrangian with $s<n$ and $n_{d f}=n-s\left(n_{d f}\right.$ : number of degrees of freedom). The general form of Eq. (1) of the two minimum conditions of the scalar potential is

$$
\begin{array}{r}
v=v(\gamma ; \beta), \quad \tan \beta=\tan \beta(\gamma, v) \\
\gamma_{\alpha}: m_{0}, \mu, A_{0}, B_{0}, M_{1}, M_{2}, M_{3}, \ldots
\end{array}
$$

leading to ${ }^{3} v=v(\gamma, \beta(\gamma))$; to simplify the notation, hereafter we refer to this dependence as $v(\gamma)$. From a Taylor series for $m_{Z} \sim v$ about a particular point $\gamma^{0}$,

$$
m_{Z}=m_{Z}\left(\gamma^{0}\right)+\left(\frac{\partial m_{Z}}{\partial \gamma_{\alpha}}\right)_{\gamma=\gamma^{0}}\left(\gamma_{\alpha}-\gamma_{\alpha}^{0}\right)+\cdots
$$

Assume for a moment that $\gamma^{0}$ is a solution ${ }^{4}$ to $m_{Z}(\gamma)=m_{Z}^{0}$, with $m_{Z}^{0} \approx 91.187 \mathrm{GeV}$. Eq. (5) can be rewritten as

$$
\begin{aligned}
\frac{m_{Z}-m_{Z}\left(\gamma^{0}\right)}{m_{Z}\left(\gamma^{0}\right)} & =\Delta n^{\alpha} \frac{\gamma_{\alpha}-\gamma_{\alpha}^{0}}{\gamma_{\alpha}^{0}}+\mathcal{O}\left(\left(\gamma_{\alpha}-\gamma_{\alpha}^{0}\right)^{2}\right), \\
n^{\alpha} & \equiv \frac{1}{\Delta}\left(\frac{\partial \ln m_{Z}}{\partial \ln \gamma_{\alpha}}\right)_{\gamma=\gamma^{0}},
\end{aligned}
$$

\footnotetext{
${ }^{2} \gamma$ can include nuisance variables (Yukawa couplings, etc.) eliminated later by integration/profiling.

${ }^{3}$ One often replaces $\beta$ by some other parameter, like $B_{0}$, with no implications below.

${ }^{4}$ Note that with an overconstrained set of parameters $\gamma_{\alpha}$ by the set of observables $\left(n_{d f}>0\right), \gamma^{0}$ above should actually denote the set that minimizes the global $\chi^{2}$ of all observables, including $m_{Z}$, rather than the solution to $m_{Z}(\gamma)=m_{Z}^{0}$ (see Sec. I. D). Then $m_{Z}\left(\gamma^{0}\right)$ does not reproduce the central measured value, but a value that should be within few standard deviations from it (say $2 \sigma_{z}$ ); then the difference in the lhs of (6) should again be understood as $2 \sigma_{z}$.
} 
where $n^{\alpha}$ denote the components of the normal to the surface $m_{Z}\left(\gamma^{0}\right)=m_{Z}^{0}$, with $n_{\alpha} n^{\alpha}=1$. Here $\Delta$ is given in Eq. (3) with Eq. (4).

For the recently measured $m_{h} \approx 126 \mathrm{GeV}$ [10], we know that minimal $\Delta$, upon varying all allowed parameters $\gamma_{\alpha}$ and $\tan \beta$, is $\Delta \sim \mathcal{O}(1000)$ [9] in the MSSM-like models with different boundary conditions for the soft terms. Note however that in the general version of the NMSSM (GNMSSM) a value of $\mathcal{O}(20)$ is still possible [11]. Then to keep $m_{Z}$ of Eq. (6) within $2 \sigma_{z}$ of its central measured value ${ }^{5}$ $m_{Z}^{0}$ or equivalently $\delta m_{Z} / m_{Z}^{0}=4.6 \times 10^{-5}$, one must keep each parameter $\gamma_{\alpha}$ within an order of $\delta \gamma_{\alpha} / \gamma_{\alpha}^{0} \approx 4.6 \times 10^{-8}$ of its value $\gamma_{\alpha}^{0}$. For simplicity, assume that all parameters other than one of them, say $\mu$, are fixed and take for example $\mu_{0} \approx 1 \mathrm{TeV}$, so it would mean $\delta \mu=46 \mathrm{keV}$. Such accuracy $\delta \mu$ or $\delta \gamma_{\alpha}$ needed to compensate the large $\Delta$ in the rhs of (6), can be reached by a fine scan of the parameter space; but a deviation by few $\mathrm{keV}$ deviates $m_{Z}$ by more than $2 \sigma_{z}$ from its measured value, if $\Delta \approx 1000$. So a good stability of the $\chi^{2}$ fit of $m_{Z}$ and a large $\Delta$, are not easily compatible (also recall that $\gamma_{\alpha}^{0}$ are (over)constrained to keep under control $\chi^{2}$ due to other observables). This problem reflects a relation of the "traditional" fine-tuning of Eq. (3) and the associated $\chi^{2}$ "cost" to fit $m_{Z}$ or other observables that depend on $v(\gamma)$. If one insists on keeping $m_{Z}$ a fixed input number (equal to $m_{Z}^{0}$ ), the problem remains because in the above discussion one replaces $m_{Z}$ by any another observable that depends on $v=v(\gamma)$. Therefore, the relation of $\Delta$ to $\chi^{2}$ is important and usually overlooked in the literature.

If relations among initial $\gamma_{\alpha}$ exist, dictated for example by UV symmetries (SU(5), etc), they can reduce ${ }^{6} \Delta[9,13]$. So a large $\Delta$ can simply be a sign of our ignorance of the UV physics, telling us that our theory of soft terms is inappropriate or incomplete. Aside from this possibility, dramatic fine-tuning of $\gamma_{\alpha}$ could be "natural" if $\gamma_{\alpha}$ are related to a fundamental constant of nature, whose accurate determination is crucial for the theory.

There are, however, limits to how much one can "fine-tune" $\gamma_{\alpha}$ in a given loop order. Indeed, $\gamma_{\alpha}^{0}$ are determined from the condition of minimizing total $\chi^{2}$ computed using a theoretical calculation of the observables in a fixed loop-order. This calculation is affected by an error from ignored higher loops (an example is the $2-3 \mathrm{GeV}$ theoretical error of the Higgs mass at two-loop [14]). So the perturbation theory alone inevitably introduces a theoretical error $\sigma_{\text {th }}$ to each $\gamma_{\alpha}^{0}$. Then only points with $\sigma_{\gamma_{\alpha}}>\sigma_{\text {th }}$ are actually relevant ${ }^{7}$; with this bound, from Eq. (6) $\Delta$ then has

\footnotetext{
${ }^{5}$ as usually done in the data fits for any observable.

${ }^{6} \Delta$ can also be reduced by "new physics" in the Higgs sector that can increase the Higgs mass $[11,12]$.

${ }^{7}$ A naive estimate of $\sigma_{\text {th }}$ can be, at three-loop order, $1 /\left(16 \pi^{2}\right)^{3}$, which is larger than $4.6 \times 10^{-8}$ mentioned. More correctly, $\sigma_{\text {th }}$ of each parameter is found numerically from the error of ignored loops in the theoretical value of the observables (such as that of $m_{h}$ mentioned) that depend on that parameter.
}

an upper bound if one insists to keep $m_{Z}$ within say $2 \sigma_{z}$ from its central value. So $\delta \chi^{2}$ and $\Delta$ are related.

The above discussion can be extended to all observables that depend on the EW scale $v(\gamma)$; then a relation between each observable and the amount of tuning of $\gamma_{\alpha}$ is present, like in Eq. (6), with a similar connection to their $\chi^{2}$ contribution. ${ }^{8}$ It is then natural to expect a more general connection between the total $\chi^{2}$ (or more generally, the likelihood) of all observables including $m_{Z}$ and their fine-tuning with respect to $\gamma_{\alpha}$. The generalisation of $\sigma_{\gamma}$ and $\sigma_{z}$ discussed above is the covariance matrix, therefore the latter could be the missing link in this connection (see later, Sec. I. D).

\section{Fixing the EW scale and the relation to $\Delta$}

Let us denote by $L(\mathcal{O} \mid \gamma)$ the total likelihood to fit some observables $\mathcal{O}_{i}$ other than $m_{Z}$. We impose on this likelihood the condition of fixing the EW scale that motivated SUSY, that we regard just as a condition to fit $m_{Z} \sim v$ of Eq. (1) to its central measured value. We first take a Dirac delta distribution for $m_{Z}$. Assuming that we can factorize this distribution ${ }^{9}$ from $L(\mathcal{O} \mid \gamma)$ of other data, the total likelihood that accounts also for fixing the EW scale is $L\left(\mathcal{O}, m_{Z}^{0} \mid \gamma\right)=L(\mathcal{O} \mid \gamma) L\left(m_{Z}^{0} \mid \gamma\right)$ with $[5,6]$

$$
L\left(m_{Z}^{0} \mid \gamma\right)=\delta\left(1-\frac{m_{Z}(\gamma)}{m_{Z}^{0}}\right)=\frac{1}{\Delta} \delta\left(n^{\alpha}\left(1-\gamma_{\alpha} / \gamma_{\alpha}^{0}\right)\right) .
$$

In the last step we used Eq. (6), with $\Delta$ as in Eq. (3). The $\operatorname{argument}$ of the Dirac $\delta$ function ensures that one must be on the surface predicted in SUSY by the minimum condition $m_{Z}\left(\gamma^{0}\right)=m_{Z}^{0}$ giving $n^{\alpha}\left(1-\gamma_{\alpha} / \gamma_{\alpha}^{0}\right)=0$, or $\gamma_{\alpha}=\gamma_{\alpha}^{0}$. For a detailed discussion and interpretation of Eq. (7) see $[5,6]{ }^{10,11}$ Further, it is illustrative to go beyond the Dirac $\delta$ used in Eq. (7), so one can take

\footnotetext{
${ }^{8}$ In this case $\gamma_{\alpha}^{0}$ will correspond to the maximal likelihood point.

${ }^{9}$ We relax this assumption in Sec. I. D.

${ }^{10}$ As a side remark, Eq. (7) can be formally integrated in one general direction (combination of $\gamma_{\alpha}$ ) and rewritten as [5]: $\tilde{L}\left(m_{Z}^{0} \mid \gamma\right)=\left.(1 / \Delta)\right|_{\gamma_{\alpha}=\gamma_{\alpha}^{0}}$. So $1 / \Delta$ that emerged is the likelihood "cost" of respecting the SUSY condition of fixing the EW scale and is part of total $L\left(\mathcal{D}, m_{Z}^{0} \mid \gamma\right)$ to fit the data that includes $m_{Z}^{0}$. A similar interpretation was noticed on phenomenological grounds in [15]. With this, one can provide a very simple estimate of an upper bound on $\Delta$. The contribution to total $\chi^{2}$ due to $m_{z}$ alone (say $\delta \chi^{2}$ ) equals, according to the last equation $\delta \chi^{2}=-2 \ln \tilde{L}=-2 \ln (1 / \Delta$ ) (under some assumptions). By demanding a "good fit" i.e. that total $\chi^{2} / n_{d f} \approx 1,\left(\chi^{2}\right.$ includes contributions from other observables $)$ one has $\Delta<\exp \left(n_{d f} / 2\right)[5,6]$, which with usual $n_{d f} \sim 10$ gives an upper value $\Delta \sim 100$. One objection to this approach is that $\tilde{L}$ is not normal which affects the goodness of the fit criterion $\chi^{2} / n_{d f} \sim 1$.

${ }^{11}$ The minimal value of $\Delta$ (with all $\gamma_{\alpha}$ allowed to vary), grows with the Higgs mass $\Delta \sim \exp \left(m_{h} / \mathrm{GeV}\right)$ (see Figs. 1-8 and 13-16 in [9], also [16]). As a result an error $\delta m_{h}=2-3 \mathrm{GeV}$ that is the theoretical uncertainty of $m_{h}$ prediction [14] brings an uncertainty factor $\approx \exp (3) \approx 20$ for $\Delta$ and accordingly for its $\delta \chi^{2}$ effect. This uncertainty means that $\Delta \approx 20$ and $\Delta \approx 400$ can be seen as equally "acceptable." Thus the results $[5,6]$ should be regarded as a general estimate rather than a strict criteria of viability.
} 


$$
L\left(m_{Z}^{0} \mid \gamma\right)=\frac{m_{Z}^{0}}{\sqrt{2 \pi} \sigma_{z}} \exp \left(-\frac{m_{Z}^{02}}{2 \sigma_{z}^{2}}\left(m_{Z} / m_{Z}^{0}-1\right)^{2}\right),
$$

which recovers the lhs of Eq. (7) when $\sigma_{z} \rightarrow 0$. From the Taylor expansion in Eq. (5) about $\gamma_{\alpha}^{0}$ one finds, to a first approximation, with $\Delta$ as in Eq. (3)

$$
\begin{aligned}
L\left(m_{Z}^{0} \mid \gamma\right) & =\frac{1}{\Delta} L(\gamma)+\cdots \\
\Rightarrow L\left(\mathcal{O}, m_{Z}^{0} \mid \gamma\right) & =\frac{1}{\Delta} L(\gamma) L(\mathcal{O} \mid \gamma)+\cdots .
\end{aligned}
$$

The first equation is similar to Eq. (7). $L(\gamma)$ is the associated normalized distribution of the output values of parameters $\gamma$; if all $\gamma$ are fixed to $\gamma^{0}$ except one of them $\left(\gamma_{\alpha}\right)$, then

$$
L(\gamma)=\frac{1}{\sqrt{2 \pi} \sigma_{\gamma_{\alpha}}} \exp \left(-\frac{1}{2 \sigma_{\gamma_{\alpha}}^{2}}\left(\gamma_{\alpha} / \gamma_{\alpha}^{0}-1\right)^{2}\right)
$$

with estimated

$$
\sigma_{\gamma_{\alpha}}=\frac{1}{\Delta} \frac{\sigma_{z}}{m_{Z}^{0}} \gamma_{\alpha}^{0} ; \quad \text { if } \sigma_{\gamma_{\alpha}} \geq \sigma_{\mathrm{th}} \Rightarrow \Delta \leq \frac{\sigma_{z}}{\sigma_{\mathrm{th}}} \frac{\gamma_{\alpha}^{0}}{m_{Z}^{0}} .
$$

$\Delta$ that emerged in (9), (11) is the sole consequence of the condition of fixing the EW scale $\left(m_{Z}=m_{Z}^{0}\right)$ that is usually associated with fine-tuning, so it is a unique, derived measure (of fine-tuning) from this constraint. $\Delta$ also relates the normalized likelihood (of $m_{Z}$ ) to the normalized Gaussian distribution of $\gamma_{\alpha}$, about the central value $\gamma_{\alpha}^{0}$; such relation is more generic (see later). Since $\Delta$ enters in the expression of $\sigma_{\gamma_{\alpha}}$, this suggests again that in more complex cases the generalization of $\sigma_{\gamma_{\alpha}}$, the error matrix, could be related to the EW fine-tuning. Finally, if one demands $\sigma_{\gamma_{\alpha}} \geq \sigma_{\text {th }}$ (from the loop-order accuracy $\sigma_{\text {th }}$ that affects $\gamma_{\alpha}^{0}$ ) an upper bound on $\Delta$ emerges in Eq. (11). This is actually a strong bound, even assuming $\sigma_{\text {th }} \sim \sigma_{z}$, then $\Delta \leq$ $\mathcal{O}(10)$ for $\mathrm{TeV}$ valued $\gamma$ 's.

\section{The general case: More observables, correlations, and fine-tuning}

So far we ignored the correlations of $m_{Z}$ with other observables (for example with the loop-corrected Higgs masses $m_{h}, m_{H}$ ) or the fact that other observables also depend on the EW scale. We include these effects to examine the relations of the total likelihood $\left(\chi^{2}\right)$ of these observables, its deviation from its maximal $\left(\min \chi^{2}\right)$ value, and of the covariance matrix, to the EW fine-tuning. The study is restricted to Gaussian distributions for observables so is equivalent to a simple $\chi^{2}$ analysis with $\chi^{2}=-2 \ln L$; the analysis can be extended to general likelihoods.

Consider the observables $\mathcal{O}_{j},(j=1,2 \ldots n)$, of experimental central values $\mathcal{O}_{j}^{0}$, and to simplify the notation we now assume that $m_{Z}$ is also one of them, $\mathcal{O}_{n}=m_{Z}$; they are functions of $\gamma_{\alpha},(\alpha=1,2, \ldots, s)$, so $\mathcal{O}_{i}=\mathcal{O}_{i}(\gamma, v(\gamma))$, with $v(\gamma)$ as in Eq. (4). The total likelihood $L(\mathcal{O} \mid \gamma)$ due to all $\mathcal{O}_{i}$ must then be maximized with respect to the SUSY parameters $\gamma_{\alpha}$. It is convenient to work with a dimensionless form of this likelihood, ${ }^{12}$

$$
\begin{aligned}
L(\mathcal{O} \mid \gamma) & =(2 \pi)^{-\frac{n}{2}}(\operatorname{det} M)^{-\frac{1}{2}} \exp \left[-1 / 2 u_{i}\left(M^{-1}\right)_{i j} u_{j}\right], \\
u_{i} & \equiv \mathcal{O}_{i} / \mathcal{O}_{i}^{0}-1,
\end{aligned}
$$

with a (dimensionless) covariance matrix $M_{i j}=\rho_{i j} \sigma_{i} \sigma_{j} /$ $\left(\mathcal{O}_{i}^{0} \mathcal{O}_{j}^{0}\right), \rho_{i j}=\rho_{j i}$ denote the correlation coefficients, with $\rho_{i i}=1$. Let $\gamma^{0}$ denote the solution of the condition to maximize $L(\mathcal{O} \mid \gamma)$. Although not appropriate for high precision numerical studies, to illustrate the main idea a Taylor expansion can be used

$$
\mathcal{O}_{i}(\gamma)=\mathcal{O}_{i}\left(\gamma^{0}\right)+\left(\gamma_{\alpha}-\gamma_{\alpha}^{0}\right)\left(\frac{d \mathcal{O}_{i}}{d \gamma_{\alpha}}\right)_{\gamma=\gamma^{0}}+\cdots,
$$

then

$$
L(\mathcal{O} \mid \gamma)=\frac{\kappa}{\Delta} L(\gamma)+\cdots,
$$

where $\kappa$ is a constant and ${ }^{13}$

$$
\begin{gathered}
\Delta \equiv\left[\operatorname{det} M \operatorname{det} \tilde{M}^{-1}\right]^{\frac{1}{2}}, \quad \tilde{M}^{-1} \equiv \mathcal{J}^{T} M^{-1} \mathcal{J}, \\
\mathcal{J}_{i \alpha} \equiv \frac{1}{\mathcal{O}_{i}^{0}}\left[\frac{d \mathcal{O}_{i}}{d \ln \gamma_{\alpha}}\right]_{\gamma=\gamma^{0}}
\end{gathered}
$$

$\tilde{M}_{i j}$ is a $s \times s$ matrix, $\mathcal{J}_{i \alpha}$ is a $n \times s$ matrix, and

$$
\begin{aligned}
L(\gamma) & \equiv(2 \pi)^{-\frac{s}{2}}(\operatorname{det} \tilde{M})^{-\frac{1}{2}} \exp \left[-1 / 2 \tilde{\gamma}_{\alpha} \tilde{M}_{\alpha \beta}^{-1} \tilde{\gamma}_{\beta}\right], \\
\tilde{\gamma}_{\alpha} & \equiv \gamma_{\alpha} / \gamma_{\alpha}^{0}-1 .
\end{aligned}
$$

$L(\gamma)$ is the normalized distribution of $\gamma_{\alpha}$ about central $\gamma_{\alpha}^{0}$ that maximizes it and contains correlations. Equation (14) has similarities to Eqs. (7), (9).

Let us examine the matrix $\tilde{M}$ and assume for simplicity that $M_{i j}$ is diagonal, then

$$
\begin{gathered}
\tilde{M}_{\alpha \beta}^{-1}=\sum_{i=1}^{n}\left\{\left(\frac{d\left(\mathcal{O}_{i} / \sigma_{i}\right)}{d \ln \gamma_{\alpha}}\right)\left(\frac{d\left(\mathcal{O}_{i} / \sigma_{i}\right)}{d \ln \gamma_{\beta}}\right)\right\}_{\gamma=\gamma^{0}}, \\
\alpha, \beta=1,2, \ldots . . s .
\end{gathered}
$$

\footnotetext{
${ }^{12}$ The "dimensionful" form of the total likelihood is $\mathcal{L}(\mathcal{O} \mid \gamma)=$ $(2 \pi)^{-n / 2}(\operatorname{det} K)^{-1 / 2} \exp \left[-1 / 2\left(\mathcal{O}_{i}-\mathcal{O}_{i}^{0}\right)\left(K^{-1}\right)_{i j}\left(\mathcal{O}_{j}-\mathcal{O}_{j}^{0}\right)\right] ; \quad K$ is the dimensionful covariance matrix, $K_{i j}=\sigma_{i} \sigma_{j} \rho_{i j} ; \rho_{i j}=\rho_{j i}$ account for correlations; $\rho_{i i}=1$. $\mathcal{L}$ is equal to $L$ used in the text up to a constant, $L(\mathcal{O} \mid \gamma)=\left|\mathcal{O}_{1}^{0} \mathcal{O}_{2}^{0} \ldots \mathcal{O}_{n}^{0}\right| \times \mathcal{L}(\mathcal{O} \mid \gamma)$.

${ }^{13}$ In a $\chi^{2}$ language: $\kappa=(2 \pi)^{-n_{d f} / 2} \exp \left(-\chi_{\min }^{2} / 2\right)$ with $\chi_{\min }^{2}=$ $u_{i}\left(\gamma^{0}\right) M_{i j}^{-1} u_{j}\left(\gamma^{0}\right)$ and $\delta \chi^{2}=\chi^{2}-\chi_{\min }^{2}$ with $\delta \chi^{2}=-2 \ln [L(\mathcal{O} \mid \gamma) /$ $\left.L\left(\mathcal{O} \mid \gamma^{0}\right)\right]=-2 \ln \left[L(\gamma) / L\left(\gamma^{0}\right)\right]$.
} 
This expression shows the relevance of the variations of $\mathcal{O}_{i}$ "normalized" to their $\sigma_{i}$, which is somewhat expected on physical grounds. Further, $\mathcal{O}_{i}$ are functions of $v(\gamma), \mathcal{O}_{i}=\mathcal{O}_{i}(\gamma, v(\gamma))$, which is relevant in establishing the relation of this matrix to the traditional fine-tuning. As a result of this dependence, the matrix $\tilde{M}^{-1}$ contains new terms,

$\tilde{M}_{\alpha \beta}^{-1}=\left.\tilde{M}_{\alpha \beta}^{-1}\right|_{v=\text { const }}+\sum_{i=1}^{s}\left\{\left(\frac{\partial \mathcal{O}_{i} / \sigma_{i}}{\partial \ln v}\right)^{2}\left(\frac{\partial \ln v}{\partial \ln \gamma_{\alpha}}\right)\left(\frac{\partial \ln v}{\partial \ln \gamma_{\beta}}\right)\right\}_{\gamma=\gamma^{0}}+\sum_{i=1}^{s}\left\{\left(\frac{\partial \mathcal{O}_{i} / \sigma_{i}}{\partial \ln v}\right)\left(\frac{\partial \ln v}{\partial \ln \gamma_{\alpha}}\right)\left(\frac{\partial \mathcal{O}_{i} / \sigma_{i}}{\partial \ln \gamma_{\beta}}\right)+(\alpha \leftrightarrow \beta)\right\}_{\gamma=\gamma^{0}}$,

which are not present in the traditional approach of numerical data fits in which $v$ is actually a constant (fixed input) [4]. So each entry $\tilde{M}_{\alpha \beta}^{-1}$ automatically contains the EW fine-tuning represented by the partial derivatives of $v$ with respect to $\gamma_{\alpha}, \gamma_{\beta}$, from all observables that depend on $v$.

This is an interesting result and suggests it is worth studying other properties of $\tilde{M}$. First, the trace

$$
\operatorname{Tr} \tilde{M}^{-1}=\sum_{i=1}^{n} \sum_{\alpha=1}^{s}\left(\frac{d \mathcal{O}_{i} / \sigma_{i}}{d \ln \gamma_{\alpha}}\right)_{\gamma=\gamma^{0}}^{2}=\sum_{i=1}^{n}\left(\frac{\partial \mathcal{O}_{i} / \sigma_{i}}{\partial \ln v}\right)_{\gamma=\gamma^{0}}^{2} \times \underbrace{\sum_{\alpha=1}^{s}\left(\frac{\partial \ln v}{\partial \ln \gamma_{\alpha}}\right)_{\gamma=\gamma^{0}}^{2}}_{\Delta^{2}}+\cdots,
$$

contains terms proportional to the traditional EW finetuning (second sum above), with contributions from all $\mathcal{O}_{i}$ that depend on $v(\gamma)$. This correction is also missed if $v$ is a constant while retaining only the explicit dependence of $\mathcal{O}_{i}$ on $\gamma$. These results can also be examined for the single observable case, such as $m_{Z}, m_{h}, m_{H}$. Being invariant under the choice of basis (of parameters), the trace has some physical meaning and then so does the EW fine-tuning that emerges from it. So $\tilde{M}$ with $v=v(\gamma)$ seems more fundamental than the fine-tuning that was introduced in the past on physical grounds. These observations are easily extended if the initial $M_{i j}$ is not diagonal.

The conclusion is that the "usual" EW fine-tuning is automatically present in the analysis of precision data fits provided that one includes the EW scale as $v=v(\gamma)$ predicted by SUSY. This result has not been investigated numerically ${ }^{14}$; one can reevaluate the precision data fits to treat $v$ as a function $v=v(\gamma)$ and include observables that depend on it $\left(m_{Z}, m_{h}\right.$, etc.) together with the additional likelihood "cost" it could bring. In this picture, the traditional EW fine-tuning per se and its numerical value may be less relevant since $\tilde{M}$ contains the information related to these.

Further, one can also consider the determinant of the (inverse) covariance matrix $\tilde{M}^{-1}$ in the basis of the fundamental parameters. It is actually more relevant to consider this determinant relative to that of the initial matrix

\footnotetext{
${ }^{14}$ It may be possible that numerical studies account for this effect in a different way. With $v\left(m_{Z}^{0}\right)$ a fixed input, the EW minimum condition brings instead a dependence say $\mu=\mu\left(\gamma_{\alpha}\right)$ where $\gamma_{\alpha}$ denote parameters other than $\mu$. With this dependence it is possible to account for the above effect, but the presence in the covariance matrix of the EW fine-tuning seen above is not manifest and is overlooked.
}

$M$, and this gives exactly $\Delta$ of Eq. (15). This factor related the normalized $L(\mathcal{O} \mid \gamma)$ and $L(\gamma)$ in Eq. (14) and it is a measure of their relative width. ${ }^{15}$ For simplicity, take the case when the number of observables $\mathcal{O}_{i}$ equals that of the parameters $\gamma_{\alpha}(n=s)$. Then,

$$
\Delta=\left(\operatorname{det} \mathcal{J}^{T} \mathcal{J}\right)^{1 / 2}
$$

In particular, for two observables (say $m_{h}$ and $m_{Z}$ ) and two parameters,

$$
\Delta=\Delta_{1} \Delta_{2}\left[1-\xi_{12}\right]^{1 / 2}
$$

where

$$
\begin{aligned}
\xi_{12} & \equiv \frac{1}{\Delta_{1}^{2} \Delta_{2}^{2}}\left[\mathcal{J}_{1 \alpha} \mathcal{J}_{2 \alpha}\right]^{2} \Delta_{k} \\
& =\left\{\sum_{\alpha}\left(\frac{\mathcal{O}_{k}\left(\gamma^{0}\right)}{\mathcal{O}_{k}^{0}} \frac{d \ln \mathcal{O}_{k}}{d \ln \gamma_{\alpha}}\right)_{\gamma=\gamma^{0}}^{2}\right\}^{1 / 2}, \quad k=1,2 .
\end{aligned}
$$

With $\mathcal{O}_{i}$ functions of $v(\gamma)$, the individual fine-tunings $\Delta_{1,2}$ of $\mathcal{O}_{1,2}$ include the EW fine-tuning and are part of this more general $\Delta$. So we see again that fine-tuning of the observables and of the EW scale is included by the covariance matrix. ${ }^{16}$ The above results answered question $Q_{2}$ in the Introduction.

\footnotetext{
${ }^{15}$ In information theory [17] $\ln \Delta$ is interpreted as the change of the differential entropy when going from a multivariate Gaussian distribution (of observables $\mathcal{O}_{i}$ ) to another one (here of parameters $\gamma_{\alpha}$ ).

${ }^{6} \Delta$ of Eq. (21) is smaller then when the variations of $\mathcal{O}_{i}$ are orthogonal $\left(\xi_{12}=0\right.$ i.e. independent $\left.\mathcal{O}_{i}\right)$.
} 
From our result above it is clear that the usual criterion of a good fit in a model $\chi^{2} / n_{d f} \approx 1\left(\chi^{2} \equiv-2 \ln L\right)$ imposed on this matrix in a numerical analysis of the EW data with $v=v(\gamma)$ should then automatically take fine- tuning into account; this can then bring bounds on EW fine-tuning. In a simplified setup and under additional assumptions, this procedure was used in $[5,6]$ to set bounds on $\Delta$. We do not pursue this method here.

In the following let us be more general and analyze instead the $s$-standard deviation confidence interval, defined by the surface, ${ }^{17}$

$$
-2 \ln L\left(\gamma^{\prime}\right) \leq-2 \ln L\left(\gamma^{0}\right)+s^{2},
$$

with $L\left(\gamma^{0}\right)=L_{\max }$ and $\gamma^{\prime}=\gamma^{\prime}(s)$. In $\chi^{2}$ language this becomes $\delta \chi^{2}=\chi^{2}-\chi_{\min }^{2} \leq s^{2}$. In our approximation this condition becomes, from Eqs. (16), (17),

$$
\begin{aligned}
\delta \chi^{2} & =\tilde{\gamma}_{\alpha} \tilde{M}_{\alpha \beta}^{-1} \tilde{\gamma}_{\beta} \\
& =\sum_{i=1}^{n}\left\{\left(\frac{d \mathcal{O}_{i} / \sigma_{i}}{d \ln \gamma_{\alpha}}\right)_{\gamma=\gamma^{0}}\left(\gamma_{\alpha}^{\prime} / \gamma_{\alpha}^{0}-1\right)\right\}^{2} \leq s^{2}
\end{aligned}
$$

(implicit sum over $\alpha$ ). With $\mathcal{O}_{i}$ a function of $v(\gamma)$, for fixed $s$ and assuming that $\left|\gamma_{\alpha}^{\prime}-\gamma_{\alpha}^{0}\right| \geq \sigma_{\mathrm{th}, \alpha}>0$ this condition brings a bound on the EW fine-tuning. We introduced ad hoc $\sigma_{\mathrm{th}, \alpha}$ as a theoretical error of computing $\gamma_{\alpha}^{0}$, from the maximal likelihood $\left(\min \chi^{2}\right)$ condition in which theoretical values of observables are affected by ignored higher loops errors $^{18}$ (including effects of the RG flow for $\gamma_{\alpha}$ these observables depend on).

From inequality (24) for one observable only $\left(m_{Z}\right)$,

$$
\Delta \leq \frac{s \sigma_{z}}{m_{Z}\left(\gamma^{0}\right)}\left|\frac{n^{\alpha}\left(\gamma_{\alpha}^{\prime}-\gamma_{\alpha}^{0}\right.}{\gamma_{\alpha}^{0}}\right|^{-1},
$$

with $\Delta$ as in Eq. (3). Equation (25) also gives, by varying each $\gamma_{\alpha}$ separately with the remaining ones fixed, then adding these results in quadrature:

$$
\Delta \leq \frac{s \sigma_{z}}{m_{Z}\left(\gamma^{0}\right)}\left\{\sum_{\alpha \geq 1}^{s}\left(\frac{\gamma_{\alpha}^{0}}{\sigma_{\mathrm{th}, \alpha}}\right)^{2}\right\}^{1 / 2} .
$$

This bound is similar to that discussed in Sec. I. B and Eq. (11) and depends on the experimental and theoretical errors. The strength of this bound depends on the values of $\sigma_{\mathrm{th}, \alpha}$ and $\sigma_{z}$ (more generally $\sigma_{i}$ of all $\mathcal{O}_{i}$ ) and can be enhanced by the presence of more observables, seeEq. (24). Finally, using this approach in precision data fits of the EW data, a plot of the lhs of Eq. (24) giving $\delta \chi^{2}$, as a function of $\Delta$, for current value of

\footnotetext{
${ }^{17}$ The value of $s$ depends on the number of degrees of free$\operatorname{dom}_{18} n_{d f}$.

${ }^{18} \mathrm{An}$ example is that of the $2-3 \mathrm{GeV}$ higher loop error mentioned in Sec. I. B for $m_{h}$.
}

the Higgs mass could illustrate the role that fine-tuning plays in deciding if a model is realistic. Bounds (25), (26) can be generalized when original $M_{i j}$ is not diagonal (i.e. when Eq. (17) is not valid anymore).

The matrix $\tilde{M}$ has another interesting feature. A large traditional EW fine-tuning, which is more a problem of supersymmetry breaking than of supersymmetry itself, can signal that our theory of soft terms $\left(\gamma_{\alpha}\right)$ is incomplete. As mentioned, relations among soft masses (such as GUT relations among the gaugino masses, etc) can reduce its value. There is then the possibility that some parameters $\gamma_{\alpha}$ could be related. Such relations can be captured by the matrix $\tilde{M}$ as off-diagonal entries. This means that properties of this matrix can help us identify the fundamental $\gamma_{\alpha}$ under the constraints of the model. Indeed, there exists the so-called "global" correlation coefficient of one such parameter $\left(\gamma_{\alpha}\right)$ with the rest, defined as

$$
\rho_{\alpha}=\sqrt{1-\left[\tilde{M}_{\alpha \alpha}\left(\tilde{M}^{-1}\right)_{\alpha \alpha}\right]^{-1}}, \quad 0 \leq \rho_{\alpha} \leq 1 .
$$

$\rho_{\alpha}$ measures the total amount of correlation between $\gamma_{\alpha}$ and all other parameters $\gamma_{\beta}(\beta \neq \alpha)$. If $\rho_{\alpha}=0$ then $\gamma_{\alpha}$ is an independent variable while if $\rho_{\alpha} \rightarrow 1$ there is full correlation of $\gamma_{\alpha}$ with one linear combination of the other parameters; this is captured by the off-diagonal terms when inverting the matrix. $\rho_{\alpha}$ could help a better understating of the SUSY breaking soft terms. In this sense $\rho_{\alpha}, \alpha=1,2 . . s$ could also be used as a new measure of EW fine-tuning defined as $\tilde{\Delta}=\max \left|\rho_{\alpha}\right|$.

Interestingly, another coefficient was discussed previously, for the correlation between pairs of parameters, $\rho_{\alpha \beta}$ [4]; this was used to define a new measure of EW finetuning as $\max \left|\rho_{\alpha \beta}\right|$, where $\rho_{\alpha \beta} \sim \tilde{M}_{\alpha \beta} /\left(\sigma_{\alpha} \sigma_{\beta}\right)$. This measure was reached on physical grounds and again supports the connection of the matrix $\tilde{M}$ to fine-tuning, emphasized here. We insist that when one computes the coefficient $\rho_{\alpha}$ as well as $\rho_{\alpha \beta}$, the EW scale $v$ can be regarded as a function of $\gamma$ 's, to reflect this original prediction of SUSY.

To conclude, a traditional frequentist analysis of the EW observables (including $m_{Z}$ ) with the constraint that $v$ is a function $v=v(\gamma)$ is a test that remains true to the original motivation of SUSY. The EW fine-tuning due to all observables is automatically captured by the covariance matrix if $v=v(\gamma)$, and in this case there may be no need to discuss $\Delta$ separately. Upper bounds on the EW fine-tuning emerge, as shown in Eq. (25), from the standard deviation interval constraint discussed (imposed on this matrix), see Eq. (23) and the ignored higher-loops error $\left(\sigma_{\mathrm{th}, \alpha}\right)$ affecting the theoretical calculation of the UV parameters $\gamma_{\alpha}$ of the Lagrangian. ${ }^{19}$

This discussion relied on a Taylor expansion of $\mathcal{O}_{i}$ to linear order which, although illustrative for our purpose, is

\footnotetext{
${ }^{19}$ Upper bounds on $\Delta$ also emerge from the criterion $\chi^{2} / n_{d f} \approx 1$ (good fit) [5,6], not discussed here.
} 
not acceptable for precision studies. A numerical approach, with $v=v(\gamma)$, can avoid this approximation.

\section{CONCLUSIONS}

Unlike the SM, its supersymmetric versions stabilize the EW scale $v \sim m_{Z}$ at the quantum level and predict that $v$ is a derived quantity, function of the SUSY UV parameters $\gamma_{\alpha}$ (soft masses, couplings and $\mu$ ), so $v=v(\gamma)$. Whether this SUSY prediction successfully recovers its experimental value is the natural test of this theory. This view remains true to the original motivation of SUSY. Past estimates showed that fixing the EW scale to its measured value affects the likelihood to fit the data by a factor related to the EW fine-tuning. Here we examined this problem in a different, more general approach.

The result is that the covariance matrix in the basis of the parameters $\gamma_{\alpha}$ automatically encodes information about the EW fine-tuning provided that the EW scale is regarded as a function $v=v(\gamma)$ (rather than a constant). Note that such connection between this matrix and the EW fine-tuning was not previously examined in the literature, even though each of these aspects were studied separately. Further, the trace of the inverse of the covariance matrix and its determinant also contain the EW fine-tuning due to all $\mathrm{EW}$ observables that depend on $v$. This indicates that the EW fine-tuning is somewhat less fundamental since this matrix includes its effects through the variations of the EW scale $v$ with respect to $\gamma_{\alpha}$, (closely related to $\Delta$ ).

A consequence of the above result is that the evaluation of the traditional EW fine-tuning per se is then less relevant for the viability of a model as long as with $v=v(\gamma)$ a good $\delta \chi^{2}$ of the observables (including $m_{Z}$ ) is still possible in that model, within the theoretical approximation (loop order) considered. From this condition and approximation one can subsequently infer numerical bounds on the EW finetuning. More explicitly, the deviation $\delta \chi^{2}$ from the minimal value $\chi_{\min }^{2}$ is affected by the EW fine-tuning; so for a $s$-standard deviation confidence interval (region) and a given theoretical error (loop order), a bound on the traditional measure of the EW fine-tuning ("in quadrature") was obtained [Eqs. (25), (26)]. This eliminates subjective criteria for "acceptable" numerical values for $\Delta$.

At present the above effect seems to be overlooked in the precision data fits in the frequentist approach where $v$ is actually a fixed, input constant, so the fine-tuning-related corrections to the covariance matrix shown in the text [Eq. (18)] seem to be ignored; or they are indeed included but in a way which does not make manifest the role of the EW fine-tuning that we showed. This effect needs further numerical investigation. Our result also answers how to compare a model with a good fit of the data but significant EW fine-tuning against a model with nearly as good a fit but less EW fine- tuning: since fine-tuning effects are included in the covariance matrix, one simply chooses the model with the best fit obtained with $v=v(\gamma)$. This answers our remaining question $\left(Q_{3}\right)$ in the Introduction.

A large EW fine-tuning can be an indication of our ignorance of the details of the SUSY breaking mechanism and of the lack of a theory of soft terms. It is known that symmetries that relate the soft terms can reduce its value. The global correlation coefficient of the covariance matrix can show if a particular parameter $\gamma_{\alpha}$ is correlated with a combination of the rest. This could help one trace the more fundamental SUSY parameters and better understand the relation of fine-tuning to supersymmetry breaking.

\section{ACKNOWLEDGMENTS}

The author thanks Stefan Pokorski for many interesting discussions. He also thanks Philip Bechtle, Werner Porod, and Tim Stefaniak for a related discussion. This work was supported by a grant from the Romanian National Authority for Scientific Research, CNCS-UEFIS-CDI, Project No. PN-II-ID-PCE-2011-3-0607, and in part by Programme "Nucleu" PN 09370102.
[1] L. Susskind, Phys. Rev. D 20, 2619 (1979); K. Wilson, as acknowledged in this paper.

[2] J. R. Ellis, K. Enqvist, D. V. Nanopoulos, and F. Zwirner, Mod. Phys. Lett. A 01, 57 (1986); R. Barbieri and G. F. Giudice, Nucl. Phys. B306, 63 (1988).

[3] G. W. Anderson and D. J. Castano, Phys. Lett. B 347, 300 (1995).

[4] P. Bechtle, T. Bringmann, K. Desch, H. Dreiner, M. Hamer, C. Hensel, M. Kramer, and N. Nguyen, J. High Energy Phys. 06 (2012) 098; K. Kowalska, S. Munir, L. Roszkowski, E. M. Sessolo, S. Trojanowski, and Y.-L. S. Tsai, Phys. Rev. D 87, 115010 (2013); A. Fowlie, M. Kazana, K. Kowalska, S. Munir, L. Roszkowski, E. M. Sessolo,
S. Trojanowski, and Y.-L. S. Tsai, Phys. Rev. D 86, 075010 (2012); C. Strege, G. Bertone, F. Feroz, M. Fornasa, R. R. de Austri, and R. Trotta, J. Cosmol. Astropart. Phys. 04 (2013) 013; G. Bertone, D. G. Cerdeno, M. Fornasa, R. Ruiz de Austri, C. Strege, and R. Trotta, J. Cosmol. Astropart. Phys. 01 (2012) 015; C. Strege, G. Bertone, D. G. Cerdeno, M. Fornasa, R. Ruiz de Austri, and R. Trotta, J. Cosmol. Astropart. Phys. 03 (2012) 030; O. Buchmueller, R. Cavanaugh, M. Citron, A. De Roeck, M. J. Dolan, J. R. Ellis, H. Flacher and S. Heinemeyer et al., Eur. Phys. J. C 72, 2243 (2012).

[5] D. Ghilencea, Nucl. Phys. B876, 16 (2013); D. Ghilencea and G. G. Ross, Nucl. Phys. B868, 65 (2013). 
[6] D. Ghilencea, Proc. Sci., Corfu2013 (2013) 034.

[7] B. C. Allanach, K. Cranmer, C. G. Lester and A. M. Weber, J. High Energy Phys. 08 (2007) 023; M. E. Cabrera, J. A. Casas, and R. Ruiz de Austri, J. High Energy Phys. 03 (2009) 075; M.E. Cabrera, J. A. Casas, and R. Ruiz d Austri, J. High Energy Phys. 05 (2010) 043; S. S. AbdusSalam, B. C. Allanach, F. Quevedo, F. Feroz, and M. Hobson, Phys. Rev. D 81, 095012 (2010).

[8] S. Fichet, Phys. Rev. D 86, 125029 (2012).

[9] D. Ghilencea, H. M. Lee, and M. Park, J. High Energy Phys. 07 (2012) 046.

[10] G. Aad et al. (ATLAS Collaboration), Phys. Lett. B 716, 1 (2012); S. Chatrchyan et al. (CMS Collaboration), Phys. Lett. B 716, 30 (2012); S. Chatrchyan et al., Report No. ATLAS-CONF-2012-162, CMS-PAS-HIG-12-045.

[11] G. G. Ross, K. Schmidt-Hoberg, and F. Staub, J. High Energy Phys. 08 (2012) 074; G. G. Ross and K. SchmidtHoberg, Nucl. Phys. B862, 710 (2012), and a similar result is found in the effective approach to GNMSSM: S. Cassel, D. M. Ghilencea, and G. G. Ross, Nucl. Phys. B825, 203 (2010).
[12] M. Carena, K. Kong, E. Ponton, and J. Zurita, Phys. Rev. D 81, 015001 (2010); I. Antoniadis, E. Dudas, D. M. Ghilencea, and P. Tziveloglou, Nucl. Phys. B831, 133 (2010); B8481 (2011); B841157 (2010); M. Carena, E. Ponton, and J. Zurita, Phys. Rev. D 82, 055025 (2010); 85035007 (2012); A. Brignole, J. A. Casas, J. R. Espinosa, and I. Navarro, Nucl. Phys. B666, 105 (2003); S. Cassel and D. M. Ghilencea, Mod. Phys. Lett. A 27, 1230003 (2012).

[13] D. Horton and G. G. Ross, Nucl. Phys. B830, 221 (2010).

[14] B. C. Allanach, Comput. Phys. Commun. 143, 305 (2002); G. Degrassi, S. Heinemeyer, W. Hollik, P. Slavich, and G. Weiglein, Eur. Phys. J. C 28, 133 (2003); S. Heinemeyer, Int. J. Mod. Phys. A 21, 2659 (2006).

[15] P. Ciafaloni and A. Strumia, Nucl. Phys. B494, 41 (1997); A. Strumia, arXiv:hep-ph/9904247.

[16] S. Cassel, D. Ghilencea, and G. G. Ross, Phys. Lett. B 687 , 214 (2010); S. Cassel, D. Ghilencea, and G. G. Ross, Nucl. Phys. B835, 110 (2010).

[17] T. Cover and J. Thomas, Elements of Information Theory (Wiley, New York, 2006). 